\title{
Noninvasive Measurements of Regional Cerebral Perfusion in Preterm and Term Neonates by Magnetic Resonance Arterial Spin Labeling
}

\author{
MARIA J. MIRANDA, KERN OLOFSSON, AND KARAM SIDAROS \\ Danish Research Centre for Magnetic Resonance (DRCMR) [M.J.M., K.O., K.S.], Department of Pediatrics [M.J.M., K.O.], Copenhagen \\ University Hospital, Hvidovre. DK-2650 Hvidovre, Denmark
}

\begin{abstract}
Magnetic resonance arterial spin labeling (ASL) at 3 Tesla has been investigated as a quantitative technique for measuring regional cerebral perfusion (RCP) in newborn infants. RCP values were measured in 49 healthy neonates: 32 preterm infants born before $34 \mathrm{wk}$ of gestation and 17 term-born neonates. Examinations were performed on unsedated infants at postmenstrual age of 39-40 wk in both groups. Due to motion, reliable data were obtained from 23 preterm and 6 term infants. Perfusion in the basal ganglia (39 and $30 \mathrm{~mL} / 100 \mathrm{~g} / \mathrm{min}$ for preterm and term neonates, respectively) was significantly higher $(p<0.0001)$ than in cortical gray matter $(19$ and $16 \mathrm{~mL} / 100 \mathrm{~g} / \mathrm{min}$ ) and white matter $(15$ and $10 \mathrm{~mL} / 100 \mathrm{~g} / \mathrm{min})$, both in preterm neonates at term-equivalent age and in term neonates. Perfusion was significantly higher $(p=0.01)$ in the preterm group than in the term infants, indicating that RCP may be influenced by developmental and postnatal ages. This study demonstrates, for the first time, that noninvasive ASL at 3T may be used to measure RCP in healthy unsedated preterm and term neonates. ASL is, therefore, a viable tool that will allow serial studies of RCP in high-risk neonates. (Pediatr Res 60: 359-363, 2006)
\end{abstract}

$\mathrm{H}^{\mathrm{s}}$ igh-risk neonates, either infants born prematurely or asphyxiated infants born at term, have a vulnerable cerebral circulation (1). Impaired autoregulation of the CBF and thus cerebral perfusion contributes to some extent to the development of brain damage in those infants (2-4). Other mechanisms, as inflammation-mediated perfusion disturbances might also be implicated in brain damage development (5), however, this point has not yet been demonstrated.

Studies of the cerebral circulation have previously been performed using different invasive methods as xenon clearance $(1,6)$, positron emission tomography (PET) $(7,8)$, and SPECT (9). Noninvasive approaches for estimating global CBF, e.g. Doppler ultrasonography and NIRS (10) have been used achieving only a limited use (11). NIRS as a cot-side method for measuring CBF was described in the late 1980s (12) but has not yet become a routine application, probably because methodological limitations are a problem in quanti-

Received October 31, 2005; accepted April 30, 2006

Correspondence: Maria Jose Miranda, M.D., Ph.D., Danish Research Centre for Magnetic Resonance (DRCMR) 340, Copenhagen University Hospital, Hvidovre. Kettegaard allé 30, DK-2650 Hvidovre, Denmark; e-mail: mariam@drcmr.dk.

This work was supported by grants from the Ludvig and Sara Elsass Foundation.

DOI: 10.1203/01.pdr.0000232785.00965.b3 tative NIRS perfusion measurements. However, quantitative measurements of $\mathrm{CBF}$ in neonates have recently been performed with sonographic flowmetry of carotid and vertebral arteries (13-16). From these studies, it is known that global $\mathrm{CBF}$ increases with PMA; however, quantitative regional brain perfusion data and the influence of postnatal age beyond the first 2 wk of life (16) are not yet available on healthy neonates.

In recent years, a noninvasive magnetic resonance (MR) method for accurately measuring regional brain perfusion has been developed (17). MR-Atrial spin labeling (ASL) is a completely noninvasive MR technique that enables accurate maps of RCP to be acquired in a few minutes. As this MR method is noninvasive and safe, even in very young infants, the measurements may be repeated, e.g. for monitoring changes in perfusion or response to treatment.

The basic principle in ASL measurements is that two images of the tissue of interest are acquired: a "tag" image in which the arterial blood has been magnetically labeled or tagged using a radiofrequency pulse and a "control" image in which the arterial blood hasn't been labeled. The difference between the two images is perfusion weighted and if the experimental parameters are carefully controlled to fulfill a number of requirements, perfusion can be quantified using an appropriate mathematical model. A description of various ASL techniques, requirements for absolute quantification, and possible sources of error can be found in a recent review (18).

The purpose of the present study was to investigate the feasibility of ASL as a method for measuring RCP in unsedated healthy preterm infants at term equivalent age and in term neonates. Furthermore, we aimed to establish quantitative RCP values, measured by ASL, in healthy preterm infants at term-equivalent age as compared with healthy term neonates. To the best of our knowledge, this is the first study where MR-ASL has been applied for measuring RCP in healthy preterm and term neonates.

Abbreviations: ASL, arterial spin labeling; BG, basal ganglia and thalami; CBF, cerebral blood flow; CSF, cerebral spinal fluid; GA, gestational age; GM, cortical gray matter; NIRS, near infrared spectroscopy; PMA, postmenstrual age; QUIPSS II, quantitative imaging of perfusion using a single subtraction, second method; RCP, regional cerebral perfusion; ROI, region of interest; SPECT, single photon emission computed tomography; WM, white matter 


\section{METHODS}

Ethics. The local ethics committee (municipality of Copenhagen) accepted the study, and informed parental consent was obtained in all cases. No sedation was used for the MR examinations.

Subjects/study population and design. Forty-nine infants were enrolled in this ASL study. As known, normal term birth occurs between 37 and $42 \mathrm{wk}$ of gestation. Thirty-two infants were born prematurely (group 1) at a median GA of $31 \mathrm{wk}$ (range, 28-34 wk), median PMA at the time of MR examination was $40 \mathrm{wk}(38-42 \mathrm{wk})$. Seventeen infants were healthy term neonates (group 2) born by elective and uneventful caesarean section at a median GA of $39 \mathrm{wk}$ (37-42 wk), median PMA at the time of MR 39 wk (38-40 wk). Consequently, infants in both groups had comparable developmental ages but different postnatal ages at the time of MR examination. Of those 49 infants (32 preterm/17 term), only 29 infants ( 23 preterm/ 6 term) were included in the final results, as the rest were excluded due to excessive motion. The median postnatal age in group 1 was 9 wk $(63 \mathrm{~d})$ and in group 2 was $2 \mathrm{~d}$. Apgar scores and umbilical cord pHs were within normal ranges for all infants. The healthy term infants were newborn infants from the nursery, born by elective cesarean section because of maternal causes as breach presentation or maternal fright. The premature infants were clinically stable throughout the neonatal period and had brain ultrasound scans without major lesions.

MR protocol. For the MR examination infants were unsedated, sleeping naturally after a feed and wrapped in blankets and a slightly deflated vacuum pillow. Padded silicone ear cups were used for noise protection. All images were acquired on a Siemens Magnetom Trio 3Tesla (T) scanner. Besides a high-resolution $\mathrm{T}_{2}$-weighted anatomical scan, we used a proximal inversion with a control for off-resonance effects (PICORE) quantitative imaging of perfusion using a single subtraction, second method (QUIPSS II) sequence (19) for ASL-perfusion MR measurements. Fourteen to 16 slices were acquired with a slice thickness of $5 \mathrm{~mm}$ and $0.5 \mathrm{~mm}$ interslice gaps. The imaging parameters were time to QUIPSS saturation $\mathrm{TI}_{1}=700 \mathrm{~ms}$, inversion time of the first slice $\mathrm{TI}_{2}=1500 \mathrm{~ms}$, increase in inversion time per slice $\Delta \mathrm{TI}_{2}$ $=55.2 \mathrm{~ms}$, TE (echo time) $/ \mathrm{TR}$ (repetition time) $=23 / 2700 \mathrm{~ms}$, FOV (field of view) $=192 \mathrm{~mm}, 64 \times 64$ matrix EPI (echo planar imaging) readout, giving an image resolution of $3 \times 3 \times 5.5 \mathrm{~mm}$. The slices were positioned axially covering the entire brain and the acquisition order was ascending (inferior to superior) to reduce the required $\mathrm{TI}_{2}$. The labeling slab was $10 \mathrm{~cm}$ thick and was positioned with a gap of $10 \mathrm{~mm}$ below the most proximal slice. The labeling slab thus covered the lower part of the head, the neck and in some cases the upper part of the chest, but not the heart. Tag and control images were acquired in an interleaved fashion with a total of 124-140 repetitions for averaging.

Postprocessing of MR-ASL data. Motion during the MR scan is a general problem with unsedated neonates and may cause severe artifacts in the acquired images, especially the perfusion-weighted ASL images. The following motion correction scheme was therefore implemented. The series of tag and control ASL images were realigned using the SPM2 (www.fil.ion. ucl.ac.uk/spm/) realignment tool (20). Periods with significant motion (more than $2 \mathrm{~mm}$ translation or $1.5^{\circ}$ rotation between successive images) were identified and the images from these periods were discarded. The remaining images were realigned again and the average tag and control images were subtracted to give a mean perfusion weighted image for each subject. A threshold of at least 40 motion-free images was set to ensure adequate signal-to-noise ratio. The resulting perfusion weighted images were then inspected visually (blinded to group) for residual motion artifacts and subjects with severe artifacts were excluded. ROIs were drawn on the control images in the BG, GM, and WM. See Figure 1for an example of such ROI. Mean perfusion values were calculated for each ROIs and each subject based on the general kinetic model for ASL described in (21). Global perfusion was also calculated. $\mathrm{T}_{1}$ of blood was assumed to be $1.5 \mathrm{~s}$ for perfusion quantification.

Statistical analysis. The calculated perfusion values for each ROIs were compared across ROIs and groups using a two-way ANOVA. $t$ Tests, corrected for multiple comparisons with a Bonferroni correction, were used to test the significance of perfusion differences between individual ROIs.

A test for homogeneity of variance (Levine's test) was performed for the three different ROIs (BG, GM, and WM) and the two groups (preterm and

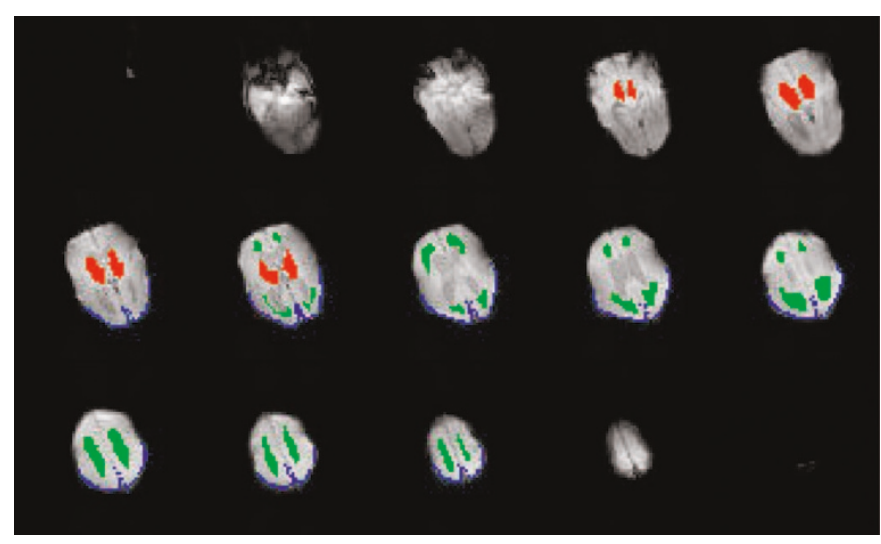

Figure 1. Axial images showing the ROIs in GM (blue), BG (red), and WM (green).

term infants) and we found that there was no statistically significant difference $(p=0.11)$ between the variances.

\section{RESULTS}

Twenty subjects were excluded due to excessive motion (9 from group 1 and 11 from group 2). For the remaining 29 subjects, 60-140 (mean, 104) images were found to be motion-free and thus contributed to the ASL images.

Figure 2 shows an example of $\mathrm{T}_{2}$-weighted anatomical images and corresponding perfusion weighted images in 12 axial slices in one subject. Regional perfusion values were calculated in three brain areas: BG, GM, and WM. The average perfusion values are listed in Table 1 and illustrated in Figure 3.

Perfusion values in the preterm infants were significantly larger than those in the term infants. The two-way ANOVA showed significant effects of both group $(p=0.011)$ and ROI $(p<0.0001)$, but no significant interactions. There were considerable differences in regional perfusion between brain areas. Perfusion was highest in the BG, with values of 39 and $30 \mathrm{~mL} / 100 \mathrm{~g} / \mathrm{min}$ for the preterm and term neonates respectively. Perfusion in BG was approximately twice the value in GM ( $p<0.0001$, corrected) and approximately 2.7 times the value in WM ( $p<0.0001$, corrected). GM perfusion was slightly larger than WM perfusion, but the difference was not significant when corrected for multiple comparisons.

Global CBF (excluding CSF) values were also calculated and were found to be $21.3 \mathrm{~mL} / 100 \mathrm{~g} / \mathrm{min}$ for preterm infants at term-equivalent age and $16.6 \mathrm{~mL} / 100 \mathrm{~g} / \mathrm{min}$ for term controls (see Table 1). The difference was, however, not quite significant ( $p=0.07)$.

ASL was found to be a feasible method for measuring perfusion in neonates even when they are unsedated. Motion is a substantial problem that can be solved in many cases.

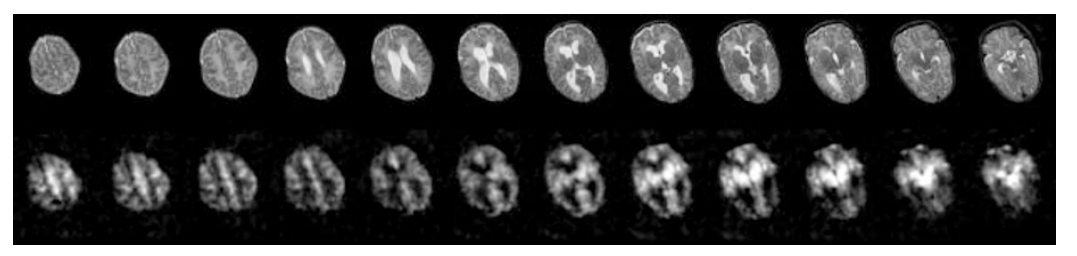

Figure 2. Anatomical $\mathrm{T}_{2}$-weighted $\mathrm{MR}$ images of a 54-d-old preterm infant scanned at term (top row) and corresponding quantitative perfusion weighted images measured noninvasively using ASL (bottom row). The perfusion weighted images were reconstructed using 112 out of 124 repetitions acquired for this subject. The images from the remaining 12 repetitions were discarded due to motion. 
Table 1. Absolute regional perfusion values for $B G, G M$, and WM as well as global perfusion measured using ASL in $\mathrm{mL} / 100 \mathrm{~g} / \mathrm{min}$

\begin{tabular}{|c|c|c|c|c|}
\hline \multirow[b]{2}{*}{ Brain region } & \multicolumn{2}{|c|}{$\begin{array}{c}\text { Term neonates } \\
n=6\end{array}$} & \multicolumn{2}{|c|}{$\begin{array}{l}\text { Preterm neonates } \\
\qquad n=23\end{array}$} \\
\hline & Mean & (SD) & Mean & (SD) \\
\hline BG & 30.2 & $(5.5)$ & 38.7 & (9.9) \\
\hline GM & 15.8 & (8.8) & 19.3 & (7.1) \\
\hline WM & 10.3 & $(4.8)$ & 14.8 & (7.3) \\
\hline Global CBF & 16.6 & (5.9) & 21.3 & (5.1) \\
\hline
\end{tabular}

Values are listed as mean over ROI and subjects and SD over subjects.

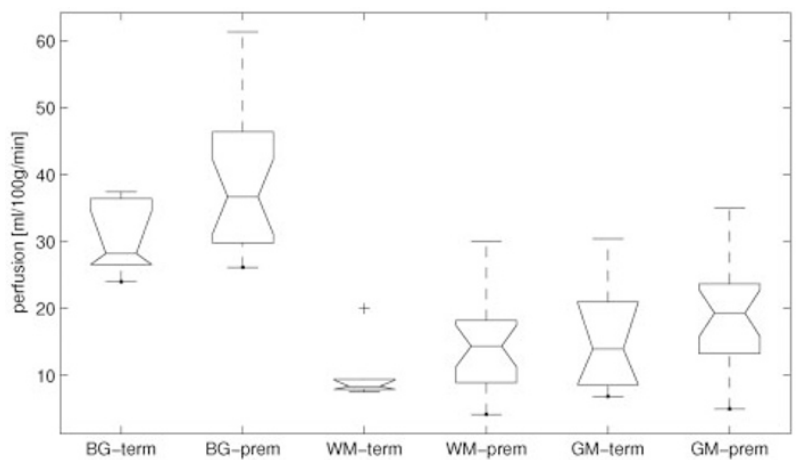

Figure 3. Box and whisker plot of the measured perfusion values (mL/100 $\mathrm{g} / \mathrm{min}$ ) in the premature and term neonates averaged over three brain regions: BG, WM, and GM.

Acquisition time is short (approximately $6 \mathrm{~min}$ ). The calculated values are comparable to $\mathrm{CBF}$ values acquired using other methods $(8,22)$.

\section{DISCUSSION}

The role of perfusion in the pathogenesis of brain damage in the high-risk neonate, either preterm born or asphyxiated term born infant, has been widely discussed $(23,24)$. While evaluating cerebral perfusion in different clinical situations, it is crucial to have reliable data on healthy preterm and term infants. Furthermore, regional measurements in different brain areas provide additional information compared with global CBF measurements. Studies on high-risk neonates have been performed more than $10 \mathrm{y}$ ago with the xenon-clearance method, providing values of global CBF (22). The xenonclearance technique has the advantage of being a cot-side method, but it involves ionizing radiation. Besides, this method does not provide regional measurements of CBF. Other methods, which involve ionizing radiation, as PET (8), Xe-CT (xenon-computed tomography) (25) and perfusion CT (26), used for quantitative estimation of CBF in neonates, have also been applied. PET (8) is able to measure regional blood flow which is a great advantage. Unfortunately, PET investigations require the drawing of a substantial volume of arterial blood to quantify CBF results, which further limits its clinical use in neonates. NIRS permits quantitative estimation of the CBF (12). In spite of being a cot-side noninvasive technique, NIRS has not accomplished the primary expectations in becoming a routine-bedside method because of methodological limitations. Furthermore, NIRS does not provide regional CBF measurements. Venous occlusion plethysmography (27) is another noninvasive method from which $\mathrm{CBF}$ can be estimated. However, problems in interpretation of results have derived in not using it in the clinical practice in neonates.

Other techniques allow nonquantitative CBF observations, and they have been applied in neonates, as SPECT (9) and Doppler ultrasonography (28); however, SPECT does not provide absolute data but only flow distribution and it has also the disadvantage of involving ionizing radiation. Doppler ultrasonography measures cerebral blood velocity and deriving quantitative data on CBF is not feasible. Nevertheless, quantitative measurements of $\mathrm{CBF}$ in neonates have recently been performed with sonographic flowmetry of carotid and vertebral arteries (14). From these studies it is known that global CBF increases with PMA; however, quantitative regional brain perfusion data and the influence of postnatal age above the first 2 wk of life (16) are not yet available.

Study design. This report is based on data from a convenience sample, as we added ASL to a series of MR methods used in a study for a different purpose.

Although two ASL measurements within the same session would have been beneficial in estimating the robustness of the method, this was not possible in the majority of cases since sedation is not a routine in our hospital when studying preterm infants in research context and the duration of the scan with unsedated neonates was therefore limited by their sleep.

The ASL study was primarily designed for 1) testing the feasibility of the ASL method and 2) for establishing ASL regional perfusion values at term/term equivalent age. A secondary aim was to compare preterm and term infants who were of same PMA/developmental age but different postnatal age. Ideally, ASL measurements at multiple time-points, e.g. within the first few days after preterm birth, at term-equivalent age, and within the first 2 mo after term would have emphasized the effect of postnatal and developmental ages in both groups. Given the noninvasiveness of ASL, multiple measurements would definitely have been feasible. However, if the inclusion criteria of the study had been that the infants came back for a second scan 2 mo after being discharged from the hospital, probably even requiring sedation in most infants at that time, the recruitment of especially healthy controls would have been extremely difficult. And since multiple scans were not deemed crucial for the primary goals of the study, scans were only performed at one time-point. Further studies are therefore needed to corroborate the secondary findings obtained at term age in both groups.

Quantification issues. Quantification of CBF using ASL is based on using a mathematical model to relate the measured signals to CBF values. The quantitative accuracy of the PICORE QUIPSS II sequence is based on some conditions that must be fulfilled. First, $\mathrm{TI}_{1}$ must be less than the temporal width of the bolus of labeled blood, and second $\mathrm{TI}_{2}-\mathrm{TI}_{1}$ must be greater than the transit delay, i.e. the time it takes blood to flow from the labeling slab to the imaging slices. Previous studies $(29,30)$ have shown that flow velocities in the basal cerebral arteries and carotid arteries are lower in neonates than in adults. It is therefore reasonable to assume that a $\mathrm{TI}_{1}$ of 700 $\mathrm{ms}$, which was optimized in adults, fulfills the abovementioned first condition in neonates as well. There are no 
direct measurements of the transit delays in neonates and the chosen value of $\mathrm{TI}_{2}$ is also based on adult studies. If $\mathrm{TI}_{2}$ is too short, this would violate the second condition in the lower slices or in tissue with very long transit delays and this is a limitation of the method. This often limits the accuracy of CBF measurements in WM. On the other hand, increasing $\mathrm{TI}_{2}$ to account for long transit delays would decrease the sensitivity, especially in the upper slices, due to $T_{1}$ relaxation of the labeled blood. To reduce the effects of potentially nonoptimal $\mathrm{TI}_{2}$, the ROIs in this study were not drawn in the lowest or uppermost slices, but were concentrated in the central slices.

Interpretation of results. Perfusion was higher in BG than in the cortex both in preterm and in term born infants. Although the resolution of the ASL images is fairly good $(3 \times$ $3 \times 5.5 \mathrm{~mm}$ ), it is not high enough to avoid partial volume effects with CSF in the cortex. This may cause underestimation of the cortex perfusion. Although there is no CSF partial volume in the $\mathrm{BG}$, there are probably some WM tracts as the internal capsule lies within the BG ROIs. Perfusion in WM is lower than in the BG and GM (14.8 and $10.3 \mathrm{~mL} / 100 \mathrm{~g} / \mathrm{min}$ in preterm and term infants, respectively). The validity of these numbers is, of course, based on the fulfillment of the conditions mentioned above in the previous section.

MRI is a noninvasive imaging technique. In this study, we have demonstrated that ASL is feasible for measuring perfusion in neonates, even when they are not sedated and certain movement is present in most cases. Motion is a substantial problem in this study, where $40 \%$ of infants had to be excluded for that reason. However, the motion problem can be solved in many cases (a great part of unsedated infants move a little) and the obtained perfusion values are comparable to values obtained using other known methods $(8,10,12,22)$.

Using PET, Altman et al. (8) found global CBF in preterm infants (PMA $=40 \mathrm{wk}$ in average) to be $5-23 \mathrm{~mL} / 100 \mathrm{~g} / \mathrm{min}$ (term born infants, $9-73 \mathrm{~mL} / 100 \mathrm{~g} / \mathrm{min}$ ). Edwards et al. (12) found global $\mathrm{CBF}$ of $14-33 \mathrm{~mL} / 100 \mathrm{~g} / \mathrm{min}$ using NIRS $(\mathrm{PMA}=31 \mathrm{wk})$ and Greisen et al. (22) found global CBF of $20 \mathrm{~mL} / 100 \mathrm{~g} / \mathrm{min}$ (PMA $=28-33 \mathrm{wk}$ ) using xenon clearance in preterm infants, too. These values are very similar to those we find in this study $(21$ and $16 \mathrm{~mL} / 100 \mathrm{~g} / \mathrm{min}$ for the preterm and term infants, respectively).

The regional $\mathrm{CBF}$ values reported here, especially for the preterm babies, are higher than the regional CBF-values measured by Altman et al. (8). However, they only calculated regional $\mathrm{CBF}$ in a little subgroup of sick infants where global $\mathrm{CBF}$ was below $10 \mathrm{~mL} / 100 \mathrm{~g} / \mathrm{min}$. Furthermore, the average postnatal age in this subgroup was $8 \mathrm{~d}$ compared with $9 \mathrm{wk}$ in our study. One might expect even lower values on the WM; however, the values we found probably reflect the high postnatal age of this group of healthy infants, compared with infants in previous studies. Our finding of relatively high WM perfusion is also reflected in a lower flow ratio between BG and WM (2.6 for preterm/3 for term infants) compared with Borch and Greisen's (9) results (4.3-5.9 for preterm infants); however, the infants in Borch and Greisen's study are younger preterm infants (median GA, $27 \mathrm{wk}$ ) examined early in the neonatal period, where perfusion in the WM is known to be very low.
It has recently been shown by ultrasound flowmetry that CBF volume increases continuously at least during the first 2 wk of life, suggesting that both gestational age and postnatal ages have an influence on CBF values. These findings support our results. The relatively high perfusion values we have found in the BG in both groups are also in concordance with the high metabolic rate in these areas (31).

ASL has the limitation of motion and that it is not a cot-side method, which in practice limits several repetitions in a short period of time. However, the advantage of ASL compared with other known methods is that it allows us to calculate perfusion in any chosen brain region. Specific differences in perfusion are very important while evaluating vulnerability or specific patterns of injury. In the future, when serial measurements are needed to evaluate a given clinical situation, sedation is an option which without doubt will improve ASL measurements considerably. The great routine and relatively easy availability of MR for examining neonates in many centers will permit serial ASL measurements in selected groups of infants.

In summary, our results are comparable to others; however, they are more consistent as our group is very homogeneous because all infants are healthy and they have comparable PMA. To the best of our knowledge, such a study has not been performed before.

Our study addresses new important knowledge about the regional perfusion of different brain areas in healthy neonates. The influence of preterm birth (GA and postnatal age) on cerebral perfusion is also addressed in this study by the fact that preterm infants at term equivalent age have higher regional perfusion values compared with healthy term born infants in the three brain areas studied. This fact confirms the knowledge from recent sonographic flowmetry studies that perfusion increases with postmenstrual $(13,14)$ and postnatal (16) ages. Preterm infants were easier to examine unsedated than term-born neonates. The latter group, who were only $2 \mathrm{~d}$ old on average at the time of scanning, were more likely to move during the procedure, even when asleep and thereby reliable measurements were more difficult to obtain in those infants.

In summary and in spite of the limitations described above, ASL is a feasible approach for measuring brain perfusion and offers the possibility of serial investigations in high-risk neonates, such as severely asphyxiated infants, and thus the opportunity of following the progression of the regional perfusion changes eventually leading to severe brain damage (23). Improving methodological aspects is, however, needed for routine application of this method in clinical settings.

Acknowledgments. The authors thank Pia Olsen for critical assistance in scanning all infants. We also thank the personnel from MR, neonatal, and nursery departments for their support and assistance, and the infants who participated and their parents for their essential involvement in this study.

\section{REFERENCES}

1. Pryds O 1991 Control of cerebral circulation in the high-risk neonate. Ann Neurol 30:321-329

2. Lou HC, Lassen NA, Friis-Hansen B 1979 Impaired autoregulation of cerebral blood flow in the distressed newborn infant. J Pediatr 94:118-121 
3. Tsuji M, Saul JP, du Plessis A, Eichenwald E, Sobh J, Crocker R, Volpe JJ 2000 Cerebral intravascular oxygenation correlates with mean arterial pressure in critically ill premature infants. Pediatrics 106:625-632

4. Greisen G 2005 Autoregulation of cerebral blood flow in newborn babies. Early Hum Dev 81:423-428

5. Yanowitz TD, Jordan JA, Gilmour CH, Towbin R, Bowen A, Roberts JM, Brozanski BS 2002 Hemodynamic disturbances in premature infants born after chorioamnionitis: association with cord blood cytokine concentrations. Pediatr Res 51:310-316

6. Greisen G, Pryds O 1988 Intravenous 133Xe clearance in preterm neonates with respiratory distress. Internal validation of $\mathrm{CBF}$ infinity as a measure of global cerebral blood flow. Scand J Clin Lab Invest 48:673-678

7. Volpe JJ, Herscovitch P, Perlman JM, Raichle ME 1983 Positron emission tomography in the newborn: extensive impairment of regional cerebral blood flow with intraventricular hemorrhage and hemorrhagic intracerebral involvement. Pediatrics 72:589-601

8. Altman DI, Powers WJ, Perlman JM, Herscovitch P, Volpe SL, Volpe JJ 1988 Cerebral blood flow requirement for brain viability in newborn infants is lower than in adults. Ann Neurol 24:218-226

9. Borch K, Greisen G 1998 Blood flow distribution in the normal human preterm brain. Pediatr Res 43:28-33

10. Skov L, Pryds O, Greisen G 1991 Estimating cerebral blood flow in newborn infants: comparison of near infrared spectroscopy and 133Xe clearance. Pediatr Res 30:570 573

11. Greisen G 1997 Cerebral blood flow and energy metabolism in the newborn. Clin Perinatol 24:531-546

12. Edwards AD, Wyatt JS, Richardson C, Delpy DT, Cope M, Reynolds EO 1988 Cotside measurement of cerebral blood flow in ill newborn infants by near infrared spectroscopy. Lancet 2:770-771

13. Kehrer M, Goelz R, Krageloh-Mann I, Schoning M 2002 Measurement of volume of cerebral blood flow in healthy preterm and term neonates with ultrasound. Lancet 360:1749-1750

14. Kehrer M, Krageloh-Mann I, Goelz R, Schoning M 2003 The development of cerebral perfusion in healthy preterm and term neonates. Neuropediatrics 34:281286

15. Kehrer M, Goelz R, Schoning M 2004 The development of haemodynamics in the extracranial cerebral arteries of healthy preterm and term neonates. Ultrasound Med Biol 30:283-287

16. Kehrer M, Blumenstock G, Ehehalt S, Goelz R, Poets C, Schoning M 2005 Development of cerebral blood flow volume in preterm neonates during the first two weeks of life. Pediatr Res 58:927-930
17. Detre JA, Leigh JS, Williams DS, Koretsky AP 1992 Perfusion imaging. Magn Reson Med 23:37-45

18. Calamante F, Thomas DL, Pell GS, Wiersma J, Turner R 1999 Measuring cerebra blood flow using magnetic resonance imaging techniques. J Cereb Blood Flow Metab 19:701-735

19. Wong EC, Buxton RB, Frank LR 1998 Quantitative imaging of perfusion using a single subtraction (QUIPSS and QUIPSS II). Magn Reson Med 39:702-708

20. Friston KJ, Ashburner J, Frith CD, Poline JB, Heather JD, Frackowiak RS 1995 Spatial registration and normalization of images. Hum Brain Mapp 3:165-189

21. Buxton RB, Frank LR, Wong EC, Siewert B, Warach S, Edelman RR 1998 A general kinetic model for quantitative perfusion imaging with arterial spin labeling. Magn Reson Med 40:383-396

22. Greisen G 1986 Cerebral blood flow in preterm infants during the first week of life Acta Paediatr Scand 75:43-51

23. Greisen G, Borch K 2001 White matter injury in the preterm neonate: the role of perfusion. Dev Neurosci 23:209-212

24. Volpe JJ, Herscovitch P, Perlman JM, Kreusser KL, Raichle ME 1985 Positron emission tomography in the asphyxiated term newborn: parasagittal impairment of cerebral blood flow. Ann Neurol 17:287-296

25. Ashwal S, Schneider S, Thompson J 1989 Xenon computed tomography measuring cerebral blood flow in the determination of brain death in children. Ann Neurol 25:539-546

26. Wintermark M, Lepori D, Cotting J, Roulet E, van Melle G, Meuli R, Maeder P, Regli L, Verdun FR, Deonna T, Schnyder P, Gudinchet F 2004 Brain perfusion in children: evolution with age assessed by quantitative perfusion computed tomography. Pediatrics 113:1642-1652

27. Cross KW, Dear PR, Hathorn MK, Hyams A, Kerslake DM, Milligan DW, Rahilly PM, Stothers JK 1979 An estimation of intracranial blood flow in the new-born infant. J Physiol 289:329-345

28. Thoresen M, Haaland K, Steen PA 1994 Cerebral Doppler and misrepresentation of flow changes. Arch Dis Child 71:F103-F106

29. Kojo M, Ogawa T, Yamada K 1996 Normal developmental changes in carotid arterial blood flow measured by Doppler flowmetry in children. Pediatr Neurol $14: 313-316$

30. Bode H, Wais U 1988 Age dependence of flow velocities in basal cerebral arteries. Arch Dis Child 63:606-611

31. Chugani HT, Phelps ME, Mazziotta JC 1987 Positron emission tomography study of human brain functional development. Ann Neurol 22:487-497 